DOI: $10.1515 /$ ausp-2015-0028

\title{
Frauenfiguren in der deutsch-jüdischen Gegenwartsliteratur. Rafael Seligmanns Roman Die jiddische Mamme
}

\author{
Alois KOMMER \\ Lehrstuhl für Angewandte Sprachwissenschaft \\ Sapientia Universität, Fakultät für Technische- und Geisteswissenschaften \\ alois.kommer@gmail.com
}

\begin{abstract}
Abstrakt. In der vorliegenden Arbeit soll der Umgang mit dem Stereotyp der jiddischen Mamme im gleichnamigen Roman des deutsch-jüdischen Schriftstellers Rafael Seligmann behandelt werden: ob - und in welchem Maß - Seligmanns Darstellung dem stereotypen Mutterbild entspricht. Begnügt er sich mit der einfachen Präsentation der Stereotypen, will er diese verwerfen oder etwa verstärken? Es geht nicht nur um „Mammes“, sondern um jüdische und nicht-jüdische Frauenfiguren, die der Stereotypisierung zum Opfer gefallen sind. Zuerst muss geklärt werden, welche Probleme das Leben für Juden und Nicht-Juden „,im Land der Täter“ aufwirft. Wenn Juden schon wegen der Niederlassung in Deutschland Gewissensbisse und Verachtung erfahren müssen, wie ist es dann möglich, über eine deutsch-jüdische Literatur zu sprechen? Und wie kommt es dazu, dass Autoren - in erster Linie Rafael Seligmann -, die überhaupt nicht in Deutschland leben dürften, sich entschlossen haben das Schweigen und die Tabus zu brechen und zu reizen?

Nach der kurzen sozialhistorischen und literaturgeschichtlichen Einordnung wird geschildert, wie das Stereotyp der ,jiddischen Mamme“ ihren Weg in die Literatur bis hin zu Seligmanns Roman gefunden hat. Frauenund Mutterfiguren des Romans werden untersucht um zu sehen, inwiefern diese dem vorhandenen Bild der Mutter und der Frau entsprechen.
\end{abstract}

Schlüsselbegriffe: deutsch-jüdische Gegenwartsliteratur, Stereotyp, jiddische Mamme.

Abstract. The paper deals the stereotype of the Yiddish mother (,,jiddise máme“) as it is treated in the novel „Die jiddische Mamme“ written by Rafael Seligmann, a Jewish journalist and writer. The aim of the analysis is to search the answer to the question whether the writer presents the stereotype or fights against it. It presents not only the stereotype of the mother but that of the Jewish and German woman, as well. The analysis demonstrates that these characters fit into the stereotypes that exist in Germany and Israel.

Keywords: contemporary German literature, stereotypes, ,jiddise máme“ 


\section{Deutsch-jüdische Gegenwartsliteratur: Definitionsversuche - sozialhistorischer und literaturhistorischer Hintergrund}

Eine Abgrenzung (innerhalb der deutschen Gegenwartsliteratur) der deutsch-jüdischen Literatur der Gegenwart ist zwar möglich, ihre Einordnung in den sozialhistorischen bzw. in den literarischen Kontext gestaltet sich umso schwieriger. Die wichtigste Frage, die im Laufe der Jahre nach dem Zweiten Weltkrieg immer in den Vordergrund geriet, war, ob man überhaupt von deutsch-jüdischer Literatur sprechen kann oder darf. Diese Problematik hat tiefgreifende Wurzeln und ist mit der Identitätssuche der in Deutschland lebenden Juden eng verbunden. Daraus folgt möglicherweise auch die mangelhafte literaturwissenschaftliche Aufarbeitung der deutsch-jüdischen Literatur. Sander Gilman schreibt dazu: „Sie (die Literaturkritiker) haben heutigen deutsch-jüdischen Schriftstellern [...] ihre Identität verweigert.“ (Wirtz 2011: 152)

Auch Dieter Lamping (1998: 20) gibt zu bedenken, dass es in Deutschland nicht selbstverständlich sei, über deutsch-jüdische Literatur zu sprechen, gegen den Begriff sei auch eine Reserve spürbar. Hans-Otto Horch und Itta Shedletzky (1992: 291 ff.) definieren die „deutsch-jüdische Literatur“ als das literarische Werk jüdischer Autoren deutscher Sprache, in dem explizit oder implizit in irgendeiner Form jüdische Substanz (im Sinne einer Auseinandersetzung mit jüdischer Tradition oder jüdischer Existenz) erkennbar ist. Diese entfalte sich bei den meisten Autoren innerhalb eines dominierenden deutschen kulturellen Bewusstseins. Anhand dieser Definition liegt die Frage nach einer nicht-deutschsprachigen jüdischer Literatur nahe: so könnte man z. B. auch von einer ungarisch-jüdischen Literatur sprechen, man denke hier nur an Literaturnobelpreisträger Imre Kertész oder auch Tibor Déry. (Abramson 2005: 302)

Die Werke, zu denen auch Rafael Seligmanns Roman Die jiddische Mamme, der den Basis der vorliegenden Arbeit bildet, gezählt wird, lassen sich unter verschiedenen Bezeichnungen in den literarischen Kanon einordnen. Forscher und Literaturwissenschaftler verwenden in ihren Werken verschiedene terminologische Bezeichnungen: manche schreiben über eine „deutsch-jüdische Literatur der zweiten Generation“ (wie z. B. Hartmut Steinecke), andere über ,junge jüdische Gegenwartsliteratur" (wie es bei Inga-Marie Kühl vorkommt), wiederum andere zählen diese Werke zu der „,jüdischen Gegenwartsliteratur in Deutschland“ (Dieter Lamping).

Für die vorliegende Arbeit werde ich für die literarische Einordnung des Romans von Seligmann die Bezeichnung „deutsch-jüdische Gegenwartsliteratur“1 verwenden, da die Erwähnung der „Generation“ möglicherweise verwirrend sein

1 Mit der Einschränkung, dass nur Werke der jüngeren Generation berücksichtigt werden. 
kann und weil ich die Meinung vertrete, dass es falsch wäre, diese - in Deutschland, in deutscher Sprache geschriebene und eindeutig auch an Nicht-Juden gerichtete - Literatur nicht als „deutsch-jüdisch“ zu bezeichnen.

\section{Sozialhistorischer Hintergrund}

Ohne Zweifel ist der Holocaust bis heute ausschlaggebend sowohl, was die Identitätsbildung der Juden in Deutschland, als auch was die nicht jüdisch-jüdischen Beziehungen angeht. Das Verhältnis wurde von Dan Diner folgendermaßen beschrieben: für Deutsche und für Juden wurde „das Ergebnis der Massenvernichtung zum Ausgangspunkt ihres Selbstverständnisses [...], eine Art von gegensätzlicher Gemeinsamkeit - ob sie es wollen oder nicht“ (Kühl 2001: 15-16).

Dass es doch eine Fortsetzung der deutsch-jüdischen Kultur gibt, ist eindeutig. Um ein Zeichen zu setzten, hat der ehemalige Vorsitzende des Zentralrates der Juden in Deutschland, Ignaz Bubis, öffentlich verlautbart, dass er Deutschland als sein Heimatland betrachte, und nannte sich einen „deutschen Staatsbürger jüdischer Herkunft“ (Kühl 2001: 37).

Nach 1945 verließen „90 Prozent der in Deutschland gestrandeten Juden [...] so schnell sie nur konnten das Land der Mörder" - schrieb Rafael Seligmann 1992 in einem Spiegel-Essay. Trotz der Behauptung, dass sie auf gepackten Koffern säßen, verließen nicht alle Juden Deutschland, viele kamen sogar zurück. Die deutsche Bundesregierung förderte ihrerseits diese Rückwanderung als Zeichen der Demokratisierung des Landes (Robertson 2004: 238). Und diejenigen, die bis zu diesem Zeitpunkt das Land der Täter nicht verlassen haben, blieben. Anfang der 90er Jahre bemerkt Seligmann, dass der „nazistische Judengenozid“ auch psychologisch erfolgreich war: „Das Angsttrauma hatte zufolge, dass die Juden sich nicht länger als Deutsche fühlten." (Seligmann 1992: 75)

In Deutschland leben heute nach Angaben des Zentralrats der Juden etwas mehr als 100000 Juden..$^{2}$ Im Falle der Holocaust-Überlebenden, der sogenannten ersten Generation, ist oft eine Tabuisierung der Katastrophe bemerkbar. Die nachfolgende Generation empfindet dieses Schweigen als beklemmend, weil, wie Eva Eichmair (2009: 58) vermutet, es die Eltern fast emotionslos erscheinen lässt - obwohl die fehlende Auseinandersetzung mit der Vergangenheit auch dadurch motiviert werden kann, die Kinder zu schützen. Die Aufarbeitung der Vergangenheit bleibt also in der Verantwortung der Nachfolgegeneration. Zudem kommt ihnen eine äußerst wichtige Rolle zugesprochen: sie sind die Hoffnungsträger (Eichmair 2009: 61). Dieser Aspekt wird in der vorliegenden Arbeit später noch angesprochen werden, da der Protagonist des Romans Die jiddische Mamme genau in diese Rolle des Hoffnungsträgers schlüpfen muss, was ihm persönlich zum Verhängnis wird.

2 Nach Angaben des Zentralrats der Juden in Deutschland, Stand Dezember 2013. 


\section{Literaturhistorischer Hintergrund}

Deutsch-jüdische Literatur wurde nach dem Holocaust als besonderer Bereich der deutschsprachigen Literatur kaum wahrgenommen und war für lange Jahre tabuisiert (Mache 2004). Kaum jemand wagte es, einen Autor mit dem Adjektiv ,jüdisch“ zu bezeichnen. Steinecke bemerkt, dass in den 80er Jahren in vielen Arbeiten das bevorstehende Ende der eigenständigen Kultur und Literatur deutschjüdischer Schriftsteller verkündet wurde (Eichmair 2009). Ausgelöst wurde diese Haltung vor allem durch das Ableben von Paul Celan und Nelly Sachs, beide wichtige Vertreter der deutsch-jüdischen Nachkriegsliteratur. Schriftsteller jüdischer Herkunft, wie z. B. Hilde Domin, Jean Améry, Wolfgang Hildesheimer haben sich nicht als jüdische Autoren verstanden (Lamping 1998). Dagegen sah der Literaturkritiker Marcel Reich-Ranicki die Hauptursache der fehlenden Werke darin, dass es in Deutschland oder in Österreich keine jüdischen Schriftsteller gäbe, ,die das Zeug zum Romanschreiben“ hätten (Seligmann 1992). Es hat vier Jahrzehnte gedauert, bis diese Tabus, diese Ängste zum Teil überwunden werden konnten.

Kühl (2001) weist darauf hin, dass auch die Verlagspolitik eine diskursbegrenzende Funktion ausübte, und nennt dafür als Beispiel Seligmanns Schwierigkeiten, seinen ersten Roman zu veröffentlichen. Die Verlagshäuser hatten u. a. befürchtet, dass das Buch antisemitische Einstellungen fördern könnte.

Seligmann selbst hält eine Auseinandersetzung mit dieser Problematik für wünschenswert, sogar für notwendig. Durch das provokante Schreiben erhalten „Juden wie Nichtjuden endlich Gelegenheit, gegen-, unter- und miteinander über die Rolle der Juden in Deutschland zu streiten" (Seligmann 1992). Er bemerkt, dass die Autoren, die zuerst „die Kraft hatten, ihre Empfindungen preiszugeben“, im Ausland, „fern der Nazifurcht“ aufgewachsen sind. Neben Irene Dische und Maxim Biller zählt er sich auch zu diesen Schriftstellern (Schmitz 2006). Seligmann selbst hat durch seine publizistische und vor allem schriftstellerische Tätigkeit vorerst kein Verständnis im Reihen der jüdischen Gemeinde gefunden. Er wurde in Bezug auf seinen Debütroman als „Nestbeschmutzer“ verhöhnt. Helene Schruff hat dies folgenderweise formuliert: ,[Seligmann] provoziert [...] Juden wie Nichtjuden mit Leidenschaft, läßt [sic!] sich dafür anstandslos als »Hoffnarr [sic!] der Gesellschaft « bezeichnen und nimmt auch die Beschimpfungen seitens der jüdischen Gemeinde als »Nestbeschmutzer« in Kauf.” (Beegle 2007: 34)

Diese Neuorientierung der deutsch-jüdischen Literatur, die Bereitschaft der jüdischen Autoren auf Konfrontationskurs zu gehen, war Mitte der 80er Jahre, also bereits vor dem Mauerfall eingetreten. Dieser Prozess wird vor allem dem in den 70er und 80er Jahren eingetretenen Generationswechsel zugeschrieben.

Bereits Mitte der 60er Jahre erhielt die deutsch-jüdische Beziehung auch in Arbeiten nichtjüdischer Autoren aus Deutschland Einzug. Diesbezüglich muss an dieser Stelle auch die literarische Fremdwahrnehmung der in Deutschland 
lebenden Juden angesprochen werden. 1967 erschien Alfred Anderschs Roman Efraim, der erste Roman eines „nicht jüdischen deutschen Autors der Nachkriegszeit, der eine jüdische Perspektive zu übernehmen versucht“ (Lamping 1998: 149). Anderschs Roman war die erste Schnittstelle dieser Art zwischen dem jüdischen und nicht-jüdischen Diskurs in der deutschen Nachkriegsliteratur. Efraim bewirkte, was auch Seligmann sich zum Ziel gesetzt hat: eine Konfrontation, eine Auseinandersetzung mit dem deutsch-jüdischen Verhältnis. Wie Dieter Lamping mit mehreren Zitaten belegt, hat der Roman Efraim eine Diskussion über die Darstellung der Juden auch unter jüdischen Kritikern ausgelöst. Eine Auseinandersetzung mit der Vergangenheit ist - mehr oder weniger explizit auch in den Werken von Ingeborg Bachmann oder Uwe Johnson vorzufinden, um nur einige weitere Beispiele zu nennen (Gehle 1998; Hofmann 1998).

Die Schriftsteller dieser Generation, z. B. Maxim Biller, Esther Dischereit, Lea Fleischmann, Barbara Honigmann, Robert Schindel, aber auch Rafael Seligmann, knüpfen jedoch nicht an die deutsch-jüdische Literatur des 19. und 20. Jahrhunderts an, wie Lamping (1998) feststellt. Seiner Ansicht nach suchen diese Autoren literarische Traditionen außerhalb der deutschen Literatur, z. B. in der jüdisch-amerikanischen - wodurch wir auch eine Erklärung für Phillip Roths Einfluss auf Seligmann hätten.

\section{Vorurteile, Stereotypen, Feindbilder - das Stereotyp der jiddischen Mamme}

Antijüdische Stereotypen und Feindbilder waren keine Erfindung der Nazis und auch kein deutsches Spezifikum. Vorurteile gegen Juden hatten sich lange Zeit vor Hitlers Machtergreifung auch im deutschsprachigen Raum verbreitet. Juden wurden oft als Sündenböcke dargestellt, für Krisen und Krankheiten verantwortlich gemacht, so wurde ihnen nach dem Ersten Weltkrieg die Schuld an der Niederlage zugeschrieben, obwohl sie zu jenem Zeitpunkt als „Deutsche“ sich hinter ihrem Land stellten und 12000 Juden ihr Leben für diesen Krieg geopfert haben (Schoeps 1992).

Antisemitismus schöpft weiterhin aus judenfeindlichen Stereotypen, die keineswegs verschwunden sind. Diese werden höchstens anders artikuliert, aber es gibt keine erkennbare qualitative Änderung, bemerkt der Antisemitismusforscher Wolfgang Benz (2007) in einem Aufsatz. Stereotype Bilder würden auch heute ihren Weg in die Medien finden.

Das Bild des Juden war und ist ein Bild, das von Vorurteilen geprägt und belastet ist. Julius Schoeps schreibt, dass dieses Zerrbild „mit der Wirklichkeit nur wenig zu tun hat, aber alle Vorurteile bündelt, die über Juden und Judentum seit jeher im Umlauf sind.“ (Schoeps 1992: 97) - angefangen beim „Gottesmörder“ 
und Hausierer, bis hin zum Stereotypen des „Blutsaugers“. Elisabeth Klamper meint, dass es in den Köpfen der Menschen bis heute ein Vorstellungssystem vorhanden ist, wie Juden zu sein haben, welche Eigenschaften, körperliche Merkmale oder Verhaltensweisen als typisch jüdisch gelten. Sie zählt auch einige solche Attribute auf: geschäftstüchtig, geizig, aufdringlich, kleinwüchsig, plattfüßig, hakennasig, feige, kraushaarig usw. (Eichmair 2009: 120)

Wenn man über Seligmanns Roman Die jiddische Mamme schreibt, kommt man am berühmten Werk des US-Amerikanischen Schriftstellers Phillip Roth Portnoy's Complaint" nicht vorbei. Viele sind der Meinung, dass Seligmann aus diesem Roman aus dem Jahr 1969 geschöpft hat. So glaubt auch Anna-Dorothea Ludewig, dass es sich bei der jiddischen Mamme um einen Abklatsch von Roth's Geschichte handelt (Ludewig 2011). Ohne auf den möglichen Einfluss von Portnoy's Complaint auf Seligmanns Roman weiter einzugehen, muss hier anlehnend an Beegle (2007) erwähnt werden, dass grundsätzliche Ähnlichkeiten zwischen den zwei Werken bestehen.

„Das Herz der Mutter ist der schönste Diamant, / Das Herz der Mutter ist der größte Schatz im Land. / Durch Wasser und durch Feuer geht eine Mutter für ihr Kind, / nichts ist ihr zu teuer, wenn nur die Kinder glücklich sind." So lautet die deutsche Version des weltbekannten Liedes aus dem Jahr 1925 A Yiddische Momme von Lew Pollack und dem Text von Jack Yellen. Ein weiteres Werk, das sich mit dem Stereotyp der jiddischen Mamme auseinandersetzt, ist Dan Greenbergs „pseudowissenschaftlicher Ratgeber [...] für praktischen Liebesterror“, mit dem Titel How to be a Jewish Mother (Gogos 2007).

„,[...] jede Mutter ist eine Terroristin, nur sind jüdische Mütter noch ein wenig mehr so." (Broder 2002: 117) Kein anderes Element hat so eine starke Symbolkraft. Die jiddische Mamme steht für Tradition, Herzensgüte, Wärme, für harmonisches Familienleben. In der jüdischen Kultur sind die Rollen klar verteilt: die Männer würden die Klappe aufreißen und die Frauen hätten das Sagen. Die Männer besitzen nur Ehrenpositionen, während die Frauen die Macht verwalten. Das sei bei den Juden schon immer so gewesen, schreibt Broder, und die Männer würden tun, was ihre Frauen wollen; diese dulden keine Widerrede. Und die Männer finden sich mit der Situation ab, nehmen sie als etwas Natürliches hin.

Die jiddische Mamme jammere über die Undankbarkeit ihrer Kinder, denen sie ihr Leben geopfert hat. Jüdische Mutterschaft sei eine Kunst, ein Geflecht raffinierter und hochentwickelter Techniken, so Broder. Die Grundlage der Beziehungen zwischen jüdischen Kindern und ihren Eltern sei die Fähigkeit „Schuldgefühle zu pflanzen, zu pflegen und zu ernten“. Schuldgefühle seien ein Mittel der Erpressung, „das die Opfer weitgehend entmachtet“ (Broder 2002: 112).

In der matriarchalen jüdischen Gesellschaft hatten jüdische Mütter seit jeher eine Schlüsselrolle. Nicht nur die Abstammung, sondern auch die kulturelle Prägung werden von den Müttern weitergegeben. In ihrem Vortrag über die Fi- 
gur der jüdischen Mutter behauptet Anna-Dorothea Ludewig, dass man im 20. Jahrhundert nur noch von einem Zerrbild der ursprünglichen jüdischen Mutter sprechen könne (Ludewig 2011). Die Hüterin des Hauses und der Tradition hat im Laufe der Zeit ihre Identität in Frage stellen müssen. Bei diesem Identitätsverlust knüpft auch Inga-Marie Kühl in ihrer Dissertation an, in der sie eine Beschreibung von Bella Goldmann vornimmt: Samys Mamme wird „durch übertriebene Fürsorglichkeit und einen sexualisierten, körperbetonten Umgang mit ihrem Sohn“ (Kühl 2001: 92) charakterisiert. Bei Holocaust-Überlebenden kann dieses Verhalten in den unbewältigten Ängsten wurzeln. Dadurch entsteht „ein mütterliches Unvermögen, die psychischen Bedürfnisse des Kindes zu befriedigen“. Kühl erklärt auf diese Weise die starke körperliche Zuwendung zu ihrem einzigen Sohn.

\section{Seligmanns Roman Die jiddische Mamme}

Nachdem er mit seinen Eltern 1957 nach Deutschland zurückgekehrt ist, lebt der Publizist, Schriftsteller, Politologe und Historiker Rafael Seligmann auch heute in Berlin, seine Autobiographie erschien 2010 unter dem aussagekräftigen Titel Deutschland wird dir gefallen.

Seligmann sei „the most radical or perhaps most reactionary“ (Beegle 2007: 32) Autor der zweiten Generation. Aus seiner publizistischen Arbeit geht hervor, dass er eine Konfrontation zwischen Juden und Nichtjuden in Deutschland für wünschenswert hält. Melissa Beegle vertritt zudem die Ansicht, dass Seligmann als erster Schriftsteller das Schweigen gebrochen hat. Zudem bemängelt sie, dass Seligmanns „revolutionäre zeitgenössische deutschsprachige jüdische Literatur“ (Beegle 2007: 47) von der Literaturwissenschaft kaum beachtet wurde.

Dieter Lamping (1998) meint, dass scharfe Kritik an Juden für die jungen deutsch-jüdischen Autoren durchaus typisch sei. Seligmann kann hierbei als Vorreiter betrachtet werden, da er in seinen Romanen sich Mühe gegeben hat ,jüdische Tabus“ zu brechen - sexuelle, religiöse und politische.

Die jiddische Mamme erschien 1990 und ist nach seinem literarischen Debüt Rubinsteins Versteigerung Seligmanns zweiter Roman. Es sei „das Frechste, das Aufsässigste, das heulend Komischste, was seit Jahren aus einer deutsch-jüdischen Feder kam“ - so wird die Rezension der Süddeutschen Zeitung auf dem hinteren Deckblatt des Romans zitiert. In seiner Zeit-Rezension hält Michael Zeller (2010) die Essenz des Romans sehr gut fest. Der ewige Student Samuel Goldmann lebt auch mit 30 bei seinen Eltern, hat weder Familie, noch einen anständigen Job. Nach zahlreichen Affären in Deutschland und Israel heiratet er durch wesentliche Mitwirkung der Mutter die sephardische Jüdin Sara. Schließlich wird er auch von seiner Frau ,aus heiterem Himmel“ (Seligmann 2000: 12) verlassen. 
Die Leser werden an diesem Punkt Teil der Geschichte, hier fängt nämlich die Erzählung an. Der Handlungsablauf ist nicht chronologisch, am Anfang des Romans wird uns ein Erlebnis aus Samuels Kindheit erzählt. Im zweiten Kapitel treffen wir den Erwachsenen Protagonisten wieder, der beim „Seelengeschäftsmann“ (JM 13) ${ }^{3}$ Rosenfeld Hilfe sucht, nachdem er von seiner Frau verlassen wurde. Doch die zwei Episoden am Anfang bilden nur die Einführung zu der eigentlichen Handlung, der praktisch Samuels Weg in die zuschnappende Ehefalle beschreibt - natürlich mit den entsprechenden Liebesstationen bei der „Nazitochter" Karin, bei seiner israelischen Tante Rebecca, bei Verena, deren Vater ein „Goj" ist, sowie bei der Israelin Margalith, die im Kibbuz lebt.

Die jiddische Mamme bietet aber mehr als nur heiratswütige Frauen und im Überfluss detaillierte Liebesspiele. Seligmanns Roman ist eine gewagte Momentaufnahme der jüdischen Gemeinschaft in Deutschland und in Israel und schreckt nicht zurück, die Schattenseiten des Lebens in Israel, in ständiger Alarmbereitschaft im fortwährenden Krieg zu verdeutlichen. Wir erhalten außerdem einen tiefen Einblick in die Denkweise der in Israel lebenden Juden, um zu entdecken, dass sie gegenüber den Schwarzen oder den Diaspora-Juden bei Weitem nicht frei von Vorurteilen und Verachtung sind. Nach Ansichten von Beegle (2007) gibt Seligmanns Die jiddische Mamme eine Erklärung dafür, wieso Juden sich für ein Leben in Deutschland nach dem Holocaust entscheiden. Die Handlung spielt zwar mehr in Israel als in Deutschland, aber genau dieser Einblick in den israelischen Alltag, mit dessen Schattenseiten, gibt zu verstehen, wieso Seligmanns Protagonisten in Deutschland leben.

Als roter Faden zieht sich das Motiv der stereotypisierten jüdischen Mutter durch den Roman. Seligmann scheut es nicht, seine „jiddische Mamme“, aber auch die anderen Frauenprotagonisten des Romans mit ausgereizten Klischees und Vorurteilen vollzupacken. Um eine stärkere Wirkung zu erlangen, wird ab und zu die Erzählperspektive gewechselt. So haben alle Frauengestalten die Möglichkeit, ihre Gedanken und Vorurteile zu verkünden. Inga-Marie Kühl (2001) deutet in ihrer Arbeit auf die grundsätzlichen Erwartungen, die Samuels Mamme als Holocaust-Überlebende an ihren Sohn stellt. Zwar sind Karin und Samy seit sieben Jahren zusammen, denkt er überhaupt nicht daran, sie zu heiraten, weil sie eine Deutsche ist. Als einziger Überlebendensohn der gesamten Familie kann sich Samuel dem „elterlichen Erwartungsdruck, eine jüdische Frau zu heiraten und Nachwuchs zu zeugen, langfristig nicht entziehen“ (Kühl 2001: 99). Zum anderen sei es ,aufgrund von Schuldgefühlen, die die Kinder hinsichtlich des schweren Schicksals ihrer Eltern ausgebildet haben“ (Kühl 2001: 99) ein Bedürfnis, den Wünschen ihrer Eltern zu entsprechen.

3 Bei weiteren Zitaten aus dem Roman Die jiddische Mamme werden in Klammern die Abkürzung JM sowie die Seitenzahl angegeben. 


\section{Vorurteile und Stereotypen im Roman}

Ob es um Diaspora-Juden, Deutsche oder die Sephardim geht, bedienen sich die jüdischen Protagonisten des Romans reichlich der Klischees und Stereotypen. Eva Eichmair stellt in diesem Bezug folgende Hypothese auf: „Möglicherweise steht hinter diesem Verfahren der Versuch, die Stereotype über die Juden durch bewusstes Darstellen anstatt von Verdrängung zu dekonstruieren." (Eichmair 2009: 125)

Für einen Betrachter erweckt Samuel den Anschein eines „Musterjuden“. Er ist Vorsitzender des Jüdischen Studentenverbandes in Deutschland, er erhält ein Stipendium und studiert in Israel weiter. Doch die Wirklichkeit ist anders: er spielt den „frommen Juden“, weil er seiner Mamme beweisen will, dass er ein guter Jude ist, obwohl er mit einer „Nazitochter“ geht. Sein ganzes Judentum, sogar die Kirche benutzt er aber nur als Vorwand: entweder um einen guten Eindruck zu machen, oder um zu fliehen.

Seine Denkweise ist selbstverständlich auch nicht frei von stereotypen Strukturen. Merkwürdigerweise hat er auch gegenüber Juden reichlich Vorurteile:

- „Kein normaler Israeli macht sich die Mühe an der Haustür zu klingeln, um anzuzeigen, daß [sic!] er da ist.“ (JM 66)

- „Die Nachbarn fragen. Nein! Dann hatte ich das ganze Haus bei mir in der Stube.“ (JM 63)

- „» ...Am gleichen Abend, an dem du mit dem Rabbi sprichst, weiß die ganze Gemeinde, daß [sic!] ich eine Schickse heiraten werde.«" (JM 16)

- „Das Sagen hatte aber die Mamme - wie in jeder jüdischen Familie.“ (JM 87)

Wir finden den Beweis auch dafür, dass Juden genauso wie die „Europäer“, Vorurteile haben. Diese Vorurteile betreffen nicht nur die Nicht-Juden, sondern auch die „Schwarzen, die zwar Juden sind aber trotzdem »anders «“; Samy nennt seine Freunde Antisemiten, weil sie Vorurteile gegenüber den „Schwarzen“ haben: Thre Vorurteile waren „übelster jüdischer Antisemitismus“ (JM 91). Samy versucht sich von diesen Vorurteilen zu entfernen, er lehnt sie ab. An dieser Stelle findet man den Beweis, dass Stereotype manchmal jeder Grundlage entbehren, meistens können sie nicht untermauert werden. Gegenüber den Europäern - besonders gegenüber Nicht-Juden - haben die meisten Juden Vorurteile: Auch die Sephardim, denn an die Stelle der ,brutalen, primitiven, aber dummen Araber waren die ausgekochten, rücksichtslosen Wus-Wus getreten“. (JM 88)

Samuel ist sich darüber im Klaren, dass er für die meisten israelischen Juden ein Deutscher ist - obwohl er von seinen Freunden in Israel angenommen wird und sie ihm nie etwas sagen. Doch er bleibt immer ein „Diasporajude“, der im „Naziland“ hockt. 
Das Stereotyp der jüdischen Mutter ist im Roman allgegenwärtig. Aber es scheint, als ob die Frauen von der Alleinherrschaft „die Schnauze voll“ (JM 226) hätten. Diesen Eindruck macht Rebecca, die es scheinbar genossen hat, dass Samuel vor der Hochzeit mit Sara sich gegen seine Mamme gerichtet hat. Vielleicht „,wünschten sie sich richtige Männer, die ihnen sagten was zu tun sei, statt ewige Kinder, die sich dauernd nach einer strengen Mamme sehnten..." (JM 226) - überlegt Samy. Margalith wäre die geeignete Person um das zu verwirklichen. Doch Samuel fehlt die Mut sich von der Mamme loszureißen: es bleibt nur bei diesem einzigen Mal, dass er es wagt, seiner Mutter „das Maul“ zu stopfen. Er gibt aber den Kampf auf, und besiegelt so sein Schicksal: er heiratet Sara, obwohl er das nicht will.

Im Roman kommt neben dem Mutter-Stereotyp auch das Bild der schönen Jüdin vor. Wie Anna-Dorothea Ludewig (2008) in einem Aufsatz zusammenfasst, ist „,die schöne Jüdin“ ein Themenkomplex, der sofort und unmittelbar eine starke Suggestivkraft entwickelt und wohl im Kopf eines jeden Bilder entstehen lässt, die in dem Spannungsfeld zwischen Hure und Heiliger angesiedelt sind, ,[...] auch immer als Projektionsfläche männlicher Phantasie, und hier wird sie, insbesondere in der zweiten Hälfte des 19. Jahrhunderts, zur femme fatale, zur erotischen Verführerin, aber auch zum rechtlosen Opfer sexueller Erniedrigung“ (Ludewig 2008: 14). Dieses Bild war auch in dem kollektiven Bewusstsein der osteuropäischen Länder, auch in Rumänien vorhanden, wie im Buch von Andrei Oişteanu (2005) zu lesen ist.

Auf das Motiv der schönen Jüdin wird vor allem im Falle der Protagonisten Bella sowie bei Rebecca, aber auch bei den anderen jüdischen Frauen angespielt. Samuel ist von der Schönheit dieser Frauen verzaubert, er kann ihnen einfach nicht widerstehen, was - wie sein Verhältnis zu seiner Tante zeigt - auch leicht zum Verhängnis werden kann.

Gewollt oder ungewollt, zeichnet Seligmann im Roman auch ein Bild der jüdischen Männer. Genauso, wie im Falle der Frauenfiguren, leben im Roman zahlreiche Männergestalten auf - die ihrerseits Stereotypen entsprechen und selber nicht frei von Vorurteilen sind, worauf hier aber nicht näher eingegangen werden kann.

\section{Die jiddische Mamme bei Seligmann}

„Ich [habe] meine Mutter in der Jiddischen Mamme und im Rubinstein viel harmloser geschildert als sie in Wirklichkeit war" - sagte der Schriftsteller selbst in einem Interview (Prangel 2003, literaturkritik.de).

Seligmann nutzt den Personen-Stereotyp (Waldemar 1982: 9) der jiddischen Mamme, um eine ganze Reihe von anderen Stereotypen zu erstellen. Er präsentiert den Lesern jüdische und nicht-jüdische Frauen, lässt seine Protagonisten selbst zu Wort kommen, dadurch können sich die Leser zur Gedankenwelt der Protagonisten - die überhaupt nicht frei von Stereotypen ist - Zugang verschaffen. 
Zwar ist der Titel des Romans klar und eindeutig, dennoch kann darüber spekuliert werden, wer mit ,jiddischer Mamme“ gemeint sei. Helene Schruff meint, dass der Titel die Person in den Mittelpunkt rückt, die in Samuel Goldmanns Leben die wichtigste Rolle spielt - die Mutter. Auf der einen Seite gäbe es hierbei gleich mehrere potentielle Mütter zur Auswahl: neben der leiblichen Mutter Bella Goldmann gibt es weitere zwei direkte Aspirantinnen: Samuels kinderlose Tante Rebecca und auch seine spätere Ehefrau, die „Schwarze“ Sara. Henrik M. Broder (2002) glaubt, dass der Titel sich gar nicht nur auf die Mutter bezieht. Eigentlich gehe es um Samuel Goldmanns Frauen.

Die besonders enge Verbindung zwischen Mamme und Jingele ist unbestreitbar. Durch die Shoa-Erfahrung wird diese Beziehung weiter verstärkt, wie Manuel Gogos in seiner Analyse des Protagonisten Samuel Goldmann feststellt:

[E]r ist auch der Sohn von Überlebenden des Holocaust. Die Mutter ist für ihn das letzte und deshalb unentbehrliche Band zu einer sich traumatisch entziehenden Vergangenheit. Die jiddische Mamme hält mit ihrer radikalen Form der Zuneigung ihre Kinder nicht nur bei der familiären Stange, sondern bindet sie so auch an die Geschichte ihres Volkes (Gogos 2007, Jüdische Allgemeine).

Diesen Faktoren ist es zu verdanken, dass die Beziehung zwischen Bella Goldmann und ihrem Sohn Samuel ungewöhnlich eng ist. Schon auf den ersten Seiten des Romans wird klar, dass hier die Grenzen der Mutter-Kind Liebe durch die körperliche Nähe überschritten werden - diese sexuelle Ladung wird später auch in Samuels Beziehung zu seiner Tante Rebecca übernommen. Bella ist die engste Vertraute ihres Sohnes, nicht einmal seine Liebesaffären sind für die Mamme ein Geheimnis. Sie geht damit über die Rolle einer passiven Vertrauten und nimmt aktiven Anteil am Leben ihres Einzelgeborenen (Kühl 2001).

\section{Frauenfiguren im Roman - Mütter, Frauen und potentielle Mütter}

Samuels verzweifelte Flucht vor den Frauen, die ihn heiraten wollen, bildet den Kern der Geschichte. Er flieht entweder vor seiner Mutter, vor seiner Tante oder vor den lästigen Freundinnen, die immer nur das Eine wollen: ihn heiraten. Aber es gibt keine Flucht, auf Druck der Mutter heiratet er die israelische Jüdin Sara - sie wird die Siegerin des nicht enden wollenden Kampfes um Samuel. Die klügste und gerissenste der Frauen darf am Ende des Romans als Braut neben Samuel stehen. Es ist nicht Karin - mit der Samuel sieben Jahre zusammen war -, weil sie, wie Bella sagt, eine „Nazitochter“ ist. Vera ist es auch nicht, weil sie nicht draufgängerisch ist, und Margalith ist es auch nicht, weil sie zu nachgiebig 
ist und nicht autoritär genug, um Samuel kontrollieren zu können. Am Ende bekommt ihn die beste Ersatz-Mamme - Sara.

Seligmann wirft das Problem der Assimilation der deutsch-jüdischen Frauen in Deutschland auf. Sie werden von den Juden meistens als Schicksen beschimpft, von den Nicht-Juden meistens auch nicht akzeptiert. In Veras Fall wird dies deutlich. Weil sie nur eine „halbe“ Jüdin ist, hat sie es bei der Partnerwahl besonders schwer: bei den echten Juden hat sie keine Chance, niemand will sie heiraten.

Helene Schruff schreibt über Bella Goldmann: „sie ist eine despotische, überprotektive Frau deren einziger Lebensinhalt die Sorge um ihren erwachsenen Sohn ist" (Schruff 2000: 64). Ihrer Meinung nach sei Bella die wichtigste Person in Samys Leben. Auf jeden Fall wird die Abhängigkeit des Sohnes von seiner Mutter deutlich. Samy selbst nennt seine Mutter eine „herrschsüchtige Mamme“, aber er hat nie den Mut seiner Mamme zu widersprechen - am Ende bedeutet dies seine Heirat.

Gleich im ersten Kapitel erscheint Bella im Sinne des Klischees als eine echte jiddische Mamme, hysterisch, autoritär, und wunderschön zugleich. Sie macht ihren drei Jahre alten Sohn klar, dass er immer auf seine Mutter hören muss, (,»Wenn ich dir etwas sage, dann hast du zu gehorchen, verstanden!«“ [JM 8]), den kleinen Samy wird von Anfang an klargemacht, dass er keinen eigenen Willen haben soll (,Du hast nichts zu wollen!“ [JM 8]), denn seine Mutter allein weiß ihn „zu beschützen“ (JM 8).

Die Herrschaft der Mutter nimmt mit dem Erwachsenwerden Samuels kein Ende. Sie mischt sich immer noch in das Leben seines Sohnes ein, sie will bestimmen, mit wem sich Samy anfreundet, was er anziehen soll, was er essen soll, und vor allem wen er heiraten soll - ,... Ich sage dir nur eins: Du wirst Sara heiraten.“ (JM 191) Sie setzt „Himmel und Hölle in Bewegung“ (JM 15), um Samy von seiner deutschen Freundin - die Nazitochter, wie sie Karin immer zu nennen pflegt - zu trennen. An Bellas Verhalten ändert Samys Aufenthalt in Israel fast nichts. Nach seiner Rückkehr nach Deutschland wird er von seiner Mamme ständig herumkommandiert. Natürlich fehlt das „ständige Gemäkel“ (JM 174) über die „dumme Schickse“ auch diesmal nicht. Obwohl Samy sich eine eigene Wohnung in München sucht, kann er sich von seiner Mutter nicht lösen: sie wäscht weiterhin seine Wäsche, er ruft sie täglich an. Die Mamme nützt jede Gelegenheit um ihrem Sohn klarzumachen, dass er von ihr abhängig ist und sie will diese Abhängigkeit verstärken: „Ohne mich wirst du zugrunde gehen...“ (JM 136), sagt sie zu Samuel, als dieser von zu Hause wegziehen will.

Man könnte sagen, Samys herrschsüchtige Mamme sei die Verkörperung des Stereotyps der jiddischen Mamme: sie spielt immer wieder die leidende Mamme, und versucht ihren Sohn durch Schuldgefühle zu kontrollieren. Sätze wie „»Du willst deine Mamme im Stich lassen, das ist alles «“ (JM 18) und „Dass du mich 
damit zugrunde richtest ist dir gleichgültig!“ (JM 18) kann man ziemlich oft von ihr hören. Selbst Samuel gibt zu, dass für ihre Mamme „die lebenslange Beherrschung ihrer Brut“" (JM 189) ausschlaggebend sei.

Ihre Worte und Taten untermauern diese Feststellung, und die Aussagen der anderen Protagonisten machen es noch deutlicher. Rebecca sagt über Bella, sie sei schon immer so „eingebildet“, „stolz“ und „hochnäsig“ gewesen. „Unsere Mamme war der einzige Mensch, der mit ihr fertig werden konnte." (JM 38) Samuel spricht auch nicht gerade liebevoll von seiner Mutter, er nennt sie „dumme Mutter-Kuh“ (JM 189).

Am deutlichsten wird Bellas Charakter, als Seligmann auch sie zu Wort kommen lässt. Man merkt schnell, dass sie überhaupt nicht frei von Vorurteilen ist, sie verachtet Saras Familie, weil sie „Schwarze“ sind, sie nennt sie primitiv, sie hätten „keine Ahnung von Hygiene“ (JM 194) und ihr Essen sei zwar koscher, aber unverdaulich. Als vor der Heirat die Mitgift der Braut besprochen werden soll, wird die Geldgier der Mamme eindeutig: Kein Jude hat kein Geld, merk dir das. Wenn er Kinder hat, dann hat er auch eine Verantwortung, dafür muß [sic!] er zahlen. Das weiß er. Außerdem können die alle feilschen, wie die Araber auf dem Basar. (JM 195)

Bella würde es bevorzugen, dass Samy die „Halbschickse“ Vera heiratet, weil sie wenigstens Geld habe. Ohne auf Saras Eltern Rücksicht zu nehmen, entscheidet sie ganz willkürlich, dass Sara und Samuel in Deutschland leben werden.

Man könnte meinen, Samuel hätte zwei Mütter, denn seine Tante Rebecca, die ,in der ganzen Familie als Hysterikerin verschrien (JM, 31) war und keine Kinder hatte, betrachtet ihren Neffen als ihr eigenes Kind. So gesehen ist es Samy unmöglich vor den „Möchtegern-Mammes“ (JM 207) zu entfliehen, denn in Israel - wo er sich vor Bella sicher fühlt - wartet Rebecca auf ihn. Ihre Beziehung ist von kleinen sexuellen Lustspielen geprägt. Das verrät schon der Untertitel des Kapitels „Rebecca“. Samy wurde schon als kleines Kind von seiner Tante verführt, was zu einem heftigen Streit der beiden Schwestern führte. Doch Riwkale, diese „schamlose Person“ - wie sie von ihrer Schwester genannt worden ist, setzt ihre Spielchen mit ihren Neffen jedes Mal, wenn Samy zur Besuch kommt, fort. Er hat natürlich nichts gegen diese Spielchen.

Rebecca verhält sich aber im Allgemeinen wie eine richtige Mamme. Insbesondere wenn es um die Zukunft ihres „Jingele“ geht. Als eine richtige Mutter versucht sie Samy mit verschiedenen Jüdinnen zusammen zu bringen und - genau wie Bella - setzt auch Rebecca alles daran, Samy von der „Nazitochter“ zu befreien. Aber auch in anderen Situationen scheint es so, als wäre Rebecca Samuels Mamme: auf dem Flughafen wird Rebeccas Benehmen von den Sicherheitsbeamten als ein besonders heftiger Anfall von Mammeliebe gesehen. 
„ [...] für mein Jingele ist mir nie etwas zu schade“ (JM 51) und Samuel weiß, wie er das ausnutzen kann: Rebecca verschafft ihm eine Wohnung in Israel, sie zahlt sogar die Mitgift zur Hochzeit. Es ist eine Rivalität zwischen den Schwestern zu erkennen, als würde Rebecca versuchen Bella zu übertreffen. Sie bringt den kleinen Samy dazu, ihr seine Liebe zu gestehen, dann erzählt sie Bella triumphierend, Samy würde sie mehr lieben als die eigene Mutter.

Rebeccas Methoden sind die einer echten Mamme: Erpressung und Auslösen von Schuldgefühlen. „Hast du deine Tante nicht mehr lieb?“ (JM 51) - fragt sie Samy, als dieser nicht gehorchen will. „Hast du keinen Funken Erbarmen mit deiner Tante?“ (JM 51) - will sie wissen, als sie erfährt, dass Samy die „Nazifreundin" nach Israel holen will.

Ihre Beziehung zu ihrem Ehemann ist von Verachtung geprägt. Sie duldet den impotenten Itzig neben sich, ihre Beziehung ist aber im Grunde genommen zerstört: „Jahrelang habe ich Nacht für Nacht neben einem gefühllosen Mann schlafen müssen“ (JM 48). Das wird bei der Hochzeit am Ende des Romans aus Rebeccas Erzählperspektive klar: „mein Mann stand einfach herum und gaffte“ (JM 237), oder „Daß [sic!] man diesem Mann alles sagen mußte [sic!]!“ (JM 237).

Rebecca hält auch nicht viel von den Schwarzen, aber sie hat nicht so viele Vorurteile gegenüber diesen „simplen“ Menschen, wie Bella. Die Deutschen dagegen hasst sie - gegenüber den Deutschen hat sie noch heftigere Vorurteile als ihre Schwester. Sie verachtet aber nicht nur die Deutschen, sondern auch Bella, weil sie immer noch im „Naziland“ lebt und vor allem weil sie Samy nicht in Israel leben lässt. Die Rivalität der beiden Figuren ist eindeutig und gut erkennbar: sie kämpfen um Samy. Rebecca versucht alles, um Samy nach Israel zu holen - wohin er ihrer Meinung nach hingehört. Bella weiß hingegen um die Gefahren, die durch den ständigen Krieg in Israel lauern, und will auf keinen Fall, dass ihrem Sohn etwas zustößt. Rebeccas Hass gegenüber den Deutschen scheint grenzenlos zu sein. Sie nennt Karin eine Nazitochter, die Deutschen sind ihrer Meinung nach ein Mördervolk.

Die Hochzeit von Sara und Samy sieht Rebecca wahrscheinlich als eine Chance, um ihre Rivalität geltend machen zu können. Scheinbar nimmt sie den Platz der Mutter ein, sie kümmert sich viel mehr um das junge Paar und die Hochzeit als Bella - die auch diesmal ihren Stolz nicht überwinden kann - mit Saras Vater in Streit geriet. „[W]ieder einmal lastete das Glück der Familie auf meinen Schultern." (JM 238) - sagt sie, als sie bemerkt, dass sie sich um die Hochzeit kümmern muss, weil niemand das macht. Damit trägt auch sie ihren Teil zur Erhaltung der Familie bei.

Wenn wir die Mutterfiguren des Romans betrachten, können wir Vicky, diese „etwa fünfundvierzigjährige Frau, mit verhärmten Zügen“ (JM 80) nicht außer Acht lassen. Saras Mutter stellt auf den ersten Blick den Gegenpol zur typischen „,jiddischen Mamme“ dar. Samuel selbst sagt, Vicky sei „keine hysterische jiddi- 
sche Mamme“ (JM 140). Wenn wir sie näher betrachten, können zu den anderen zwei Mutterfiguren große Unterschiede festgestellt werden.

Vicky selbst gibt zu, eine einfache Frau zu sein, sie konnte nur einige Jahre zur Schule gehen, danach musste sie zusammen mit ihrer Familie flüchten. Für diese bescheidene, gütige Frau ist die Familie, das „häusliche Glück“ am wichtigsten. Dass Samy zu Sara zurückkehrt und sie heiratet, ist auch Vickys Verdienst. Als Samy - der Sara schon verlassen hat - ihr ein Physikbuch als Geschenk bringt, nutzt „Saras ungebildete Mamme“ (JM 142) die Möglichkeit, die beiden wieder zusammen zu bringen: „Sie war freundlich, als ob ich nichts angestellt hätte. Keine Spur von Vorwürfen oder Gewissensreitereien.“ (JM 139). Sie erkennt die Vorteile einer Ehe mit Samuel und will, dass Samy - den sie liebevoll ihren Sohn nennt - und Sara zusammenbleiben und heiraten, aber sie drängt ihn nicht zu einer Entscheidung. Dennoch ist sie entschlossen, und Samy muss zugeben, dass Vicky ihm taktisch überlegen ist. Klug und gerissen überzeugt sie Samuel, und überredet ihn zur Heirat. Als die Heirat und damit auch das Glück ihrer Tochter gefährdet sind, behält sie einen klaren Kopf und setzt ihren Willen gegen ihren Mann durch. Dabei wird noch einmal deutlich, dass eigentlich Vicky im Haus das Sagen hat, sie schafft es aber, ihren Willen so geschickt durchzusetzen, dass die Ehre ihres Mannes dabei nicht verletzt wird.

Sie ist in vieler Hinsicht den zwei Mammen - Bella und Rebecca - überlegen. Sie gibt zu, kein Freund der „Wus-Wus“4 zu sein. Aber sie sagt kein schlechtes Wort über die „Europäer“ - vor allem weil sie die Heirat will, obwohl sie sehr traurig über deren Verhalten ist.

„Karin war eine Schickse, ihr Vater ehemaliger Nazi“ (JM, 15) - so lernen wir sie gleich am Anfang des Romans kennen. Nicht irgendjemand sagt diesen Satz, sondern Samuel, der seit sieben Jahren mit ihr zusammen ist. Auch hier wird der Einfluss der Mutter deutlich: Samuel benutzt die Wortwahl seiner Mutter, wenn er von Karin spricht. Karin macht überhaupt kein Geheimnis daraus, dass sie ihn heiraten will. Ganz „skrupellos“ will sie Kinder von Samuel, ohne zu berücksichtigen, dass die „Nazi-Enkelkinder“ den sicheren Tod seiner Mamme bedeuten würden. Karin gesteht, dass sie ihn liebt und mit Samuel zusammenbleiben möchte, aber sie weiß, dass Samy - aus Angst vor der Heirat, aber vor allem aus Angst vor der eigenen Mamme - überhaupt nicht daran denkt, sie zu heiraten. Trotzdem hofft sie, dass Samuel eines Tages sich von seiner Mutter lösen wird: „,...] er konnte sich doch nicht ewig unter dem Rock seiner Mutter verstecken“ (JM 76). Sie ist sogar bereit die jüdische Religion zu übernehmen, um Samuels Frau zu werden.

Es stellt sich die Frage, warum eigentlich Samy zu dieser ,wild gewordene[n] Schickse“ (JM 17) hält? Denn seine ganze Familie ist gegen die Beziehung, vor

4 Juden aus arabischen und südeuropäischen Ländern gebrauchen diesen Kosenamen für die Juden aus Mittel- und Südosteuropa. Siehe dazu den Glossar auf S. 246-247 des Romans. 
allem seine „herrschsüchtige Mamme“ - vor der er noch mehr Angst hat als vor Karin. Wenn wir dieses Problem näher betrachten, können wir feststellen, dass es einen einzigen Grund gibt, warum Karin nicht Samys Frau werden kann: sie ist eine Nicht-Jüdin. Und Bella würde es nie zulassen, dass ihr „Jingele“ eine „Nazitochter" heiratet. Nur eine Jüdin kann als Frau ihres Sohnes in Frage kommen. Samy gibt zu, dass er sich bei Karin „geborgen und geliebt“ (JM 65) fühlt, er sagt sogar, er würde „Karin erfinden, wenn es sie nicht gäbe“ (JM 119), Karin wäre eigentlich der perfekte Mutterersatz und Samy weiß das auch. Aber er kann seiner Mamme nicht die Stirn bieten.

Sara, die schöne, scheinbar unerreichbare „Schwarze“ brachte Samy die sexuelle Befreiung: „Endlich war die Liebe kein Preis mehr für die Lust wie bei meiner Mamme, bei Rebecca und bei Kara“ (JM 87). Samuel suchte nur eine Frau, als Karin nach Deutschland zurückflog, und er nicht im Bett seiner Tante landen wollte - denn nichts fürchtete er mehr, als „ohne liebende Frau zu vegetieren“ (JM 104). Sara war eben ,eine Klassefrau, nicht eine europäische, hysterisch-heulende Klette“ (JM 141). Doch ihr geht es nicht nur ums Vergnügen, Sara hat große Pläne mit Samy: sie will ihn heiraten. Und Sara hält an ihrer „Doppelmoral“ fest, nicht nur was ihr Liebesleben betrifft: sie plant von Anfang an die Heirat mit Samy, aber sagt ihm immer wieder, dass sie nicht heiraten will: „du musst dich nicht fürchten, ich werde dich nicht heiraten." (JM 173). Erst als sie in Deutschland auftaucht, gibt sie offen zu: „Meine Samuela. Ich bin hergekommen um dich zu heiraten." (JM 180).

Sara kann auch als Ersatzmamme gesehen werden. Die kluge Sara durchschaut Samuel, sie kennt seine Feigheit, seine Angst. Sie weiß ganz genau, dass sie ihr Ziel erreichen kann, obwohl Samy sich verzweifelt gegen eine Heirat wehrt. Ihr Vorteil - vor allem in den Augen der Mutter - gegenüber Karin ist, dass sie Jüdin ist, sogar eine israelische Jüdin. Deshalb gewinnt sie auch Bella für ihren Plan, und Samy ist gegen die gemeinsame Kraft seiner Mamme und der „militanten israelischen Heiratskriegerin“ (JM 185) machtlos.

Sara wird von Samy als Ersatzmamme akzeptiert, das wird aus seiner letzten Aussage klar: ,Ja, Mamme.“ (JM 245), sagt er zu seiner Braut. Und das ist genau das, was Sara will, denn „Fanatiker und Sturköpfe“ gibt es in Israel genug. Deshalb will sie den „weichen, albernen Diaspora-Samuela“ (JM 245).

Samy hat nur eine kurze Affäre mit Vera, ihre Figur ist trotzdem wichtig, weil sie einen Übergang zwischen Karin - die „Nazitochter“ und Sara - die israelische Jüdin darstellt. Sie ist nur eine „halbe“ Jüdin, ihr Vater ein reicher „Goj“ - so Samuels Mutter. Samy nimmt die Beziehung zu Vera überhaupt nicht ernst. Sie ist nur der Vorwand, um mit Sara Schluss zu machen. Vera tat schon für ihren früheren Freund Schlojme alles, und Samy will ihre mütterliche Fürsorge ausnutzen. Er denkt daran, dass Vera ihn vielleicht heiraten will, aber er macht sich darüber keine Sorgen - er will nur mit ihr schlafen. Er fühlt sich bei ihr geborgen 
und wohlig, er hat sich „zuletzt als Kind bei Bella und Rebecca“ (JM 109) so gefühlt - schon wieder wird eine Frau an der Mutter gemessen. Verena verhält sich auffallend mütterlich: zu Schlojme hat sie nur gehalten, weil sie ihm etwas geben konnte, sie konnte ihm helfen, ,auch wenn er ein Schwächling war“ (JM 121). Aber nicht nur ihre mütterliche Fürsorge und Zuneigung machen aus ihr eine potentielle jiddische Mamme. Als Samy sie rauswerfen will, versucht sie ihn durch Schuldgefühle erpressen: sie weint die ganze Nacht.

Vera ist gezeichnet von der Beziehung zu ihren Eltern, die Beziehung zu ihrer Mamme - ein ,arrogante[s], unangreifbare[s] Judenweib“ (JM 123) prägt sie besonders. Zugleich vergöttert sie ihre Mamme auch, wie wir erkennen können, als Seligmann auch Verena im Roman zu Wort kommen lässt. Veras Mutter sagt immer wieder zu ihr, dass sie eine Schickse ist, weshalb sie nie einen richtigen jüdischen Mann finden können wird; aber einem Diasporajuden, wie Samy, kann sie schon etwas bieten.

Vera erkennt selbst, dass sie den jüdischen Männern hinterher läuft, weil ihr Vater ein Goj ist und sie einen „richtigen Mann“ - also einen Juden - heiraten will. Aber gerade weil sie scheinbar leicht zu kriegen ist, wird sie von allen verachtet, sogar Karin nennt sie eine „Witzfigur“.

Es ist interessant zu beobachten wie die Beziehung zu ihrer Mutter Veras Charakter bestimmt. Gewohnt an der Herrschsüchtigkeit der Mutter, kann sie - genauso wie Samuel, keine selbstständigen Entscheidungen treffen. Ihr Charakter ist in dieser Hinsicht dem von Samuel sehr ähnlich.

„Marga hatte mich lieb - aus. Nie stellte sie Forderungen.“ (JM 158). Man sollte meinen, sie sei die ideale Frau für Samuel. Obwohl er auch weiß, dass er mit ihr eher glücklich werden könnte als mit Sara, hat ihre Beziehung keine Zukunft, weil Samuel nicht den Mut hat, zu Margalith zu stehen, er kann der „Heiratsfalle" nicht mehr entkommen. Margalith dagegen will Samy nicht beeinflussen, sie glaubt, er sei erwachsen genug, um selbst über sein Leben entscheiden zu können. Aber Samuel kann keine Entscheidungen treffen, er erwartet dass ihm gesagt wird, was er machen soll. Und Marga tut das nicht. Sie will nicht um ihn kämpfen, denn, so sagt sie, dann wäre sie auch nicht besser, als die anderen Frauen. Sie nimmt es einfach hin, dass Samy die Beziehung zu Sara fortsetzt, als wäre nichts zwischen ihnen.

Am Hochzeitstag treffen sich die beiden noch einmal: Margalith hat auch diesmal nicht den Mut - oder die Kraft - Samy zu beeinflussen. Sie weist ihn nur darauf hin, dass er mit Sara nicht glücklich sein wird. „, Du bist ein Waschlappen Shmuel."“(JM 222) - sagt sie zu ihm, als ihr klar wird, dass Samy nichts weiter ist als eine Marionette seiner „Mütter“.

Margaliths Haltung ist sehr untypisch: obwohl sie eine israelische Jüdin ist, ist sie weit von dem Bild der jiddischen Mamme entfernt. Meiner Meinung nach ist sie eine Schlüsselfigur im Roman, weil sie als Gegenpol der jiddischen Mamme 
betrachtet werden kann. Sie scheint die Einzige zu sein, die sich wirklich um Samy kümmert, sie will, dass es ihm gut geht, dass er glücklich ist. Für sie ist Samuel wichtig, seine Gefühle, sein Wille, seine Entscheidungen. Als wolle sie der Herrschaft der Mammes ein Ende setzen und den Männern klar machen, dass sie nicht immer nur das machen müssen, was man ihnen sagt. Genau deswegen - weil sie es ablehnt eine Mamme zu sein - verliert sie Samuel.

\section{Schlussbetrachtungen}

Seine Mamme war in Wirklichkeit nicht so harmlos, wie in seinen ersten Romanen dargestellt, hat Seligmann in einem Interview behauptet. Bereits aus dieser Äußerung kann man mehrere Schlüsse bezüglich des Stereotyps der ,,jiddischen Mamme“ ziehen. Erstens scheint Seligmann nichts gegen die Stereotypisierung zu haben, oder er nimmt es einfach hin, dass die Mutter- und Frauenfiguren einem festgelegten Bild entsprechen müssen.

So betrachtet wäre es einfach den jüdischen Frauen den Mamme-Stereotyp zuzuschreiben. Doch aus dem Roman und aus den Betrachtungen dieser Arbeit geht hervor, dass zum einen auch jüdische Frauen dem Mamme-Bild widersprechen können (wie bei Margalith), zum anderen aber Nicht-Jüdinnen auch MammeEigenschaften aufweisen können (wie bei Karin vermutet).

Mit Hilfe seiner Protagonisten versucht Seligmann sich gegen das Stereotyp der jiddischen Mamme zu stellen. Schicksen können genauso hysterisch und herrschsüchtig sein wie israelische Jüdinnen. Auf der anderen Seite gibt es aber auch Jüdinnen, die sich bewusst nicht als eine Mamme verhalten wollen.

Samuel Goldmann scheint sich von den Stereotypen entfernen zu wollen, er lehnt sie eindeutig ab. Er erkennt, dass nicht nur die Männer, sondern auch die jüdischen Frauen das typische Mamme-Bild satt haben. Der Schluss des Romans kann überraschend wirken: Samuel gibt den Kampf in der letzten Minute auf: „Ja, Mamme.“ - sagt er zu Sara, und dieser Satz zeigt eindeutig, dass er sich aus dem Bann der jiddischen Mamme - und von den dominierenden Stereotypen nicht lösen kann. Seine Wahl ist nicht durch den Aspekt der Kompensation von Muttersehnsucht bestimmt, entscheidend ist der Aspekt des Jüdisch-Seins bzw. Nicht-Jüdisch-Seins bei der Partnerwahl.

Was die judenfeindlichen Stereotypen angeht, macht Seligmann auch hier deutlich, dass es ein Fehler wäre zu behaupten, dass alle Deutschen judenfeindlich seien und alle Juden die Deutschen verachten würden. Im Roman kommen auch judenfeindliche Juden vor, die „Schwarze“ nicht leiden können, aber auch Juden, die alle Deutsche für Nazis halten. Ich erlaube mir zum Schluss noch eine Bemerkung, was die Legitimität Seligmanns betrifft. Man könnte ja die Frage stellen, ob Seligmann berechtigt sei, zur Provokation anzustiften. Walter Schmitz 
schreibt dazu, dass Seligmann ,gewiss nicht der Sprecher der Juden in Deutschland [ist], aber es gilt doch, dass er den Deutschen und auch den Juden etwas zu sagen hat." (Schmitz 2006: 404)

Wenn wir über den Roman Die jiddische Mamme hinwegschauen, muss hier noch erwähnt werden, dass Seligmann auch in seinen anderen Romanen, mehr oder weniger gezielt, sich mit dem Bild der Frauen beschäftigt. Das Stereotyp der jüdischen Mutter ist in Rubinsteins Versteigerung ebenso vorhanden wie im Roman Der Musterjude. Das Thema Frauenfiguren kann demnach problemlos auf das literarische Gesamtwerk von Rafael Seligmann erweitert werden. Diese Untersuchung soll aber das Thema einer nächsten Arbeit sein.

\section{Literaturverzeichnis}

Abramson, Glenda (Hrsg.) 2005. Encyclopedia of Modern Jewish Culture. London-New York, Routledge.

Beegle, Melissa. 2007. Rafael Seligmann and the German-Jewish negative symbiosis in post-shoah Germany: breaking the silence. Magisterarbeit, Bowling Green State University.

Benz, Wolfgang. 2007. Was ist Antisemitismus? Begriff und aktuelle Erscheinungsformen aus der Perspektive der internationalen Forschung. Policy. Politische Akademie. 21. http://library.fes.de/pdf-files/akademie/berlin/05053.pdf (14. Juni 2014)

Broder, Henryk M. 2002. Die jiddische Mamme. In: Mannheimer, Olga-Presser, Ellen (Hrsg.): Nur wenn ich lache. Neue jüdische Prosa... München, DTV, 106116.

Eichmair, Eva. 2009. Zwischen Schtetl, Shoa und Erez Israel: Konstruktionen jüdischer Identität in drei Werken deutsch-jüdischer Autoren der „Zweiten Generation“. Wien, Universität.

Fuß, Holger: Wer hat Israel gegründet? Interview mit Rafael Seligmann. Berliner Zeitung. http://www.berliner-zeitung.de/archiv/interview-mit-demschriftsteller-rafael-seligmann-ueber-judentum-und-antisemitismus--assimilation-und-diverse-befangenheiten-in-deutschland-wer-hat-israel-gegruendet-,10810590,9742160.html (14. Juni 2014)

Gehle, Holger. 1998. »Auschwitz« in der Prosa Ingeborg Bachmanns. In: Braese, Stephan-Gehle, Holger-Kiesel, Doron-Loewy, Hanno (Hrsg.): Deutsche Nachkriegsliteratur und der Holocaust. Frankfurt am Main-New York: Campus, 183-195.

Gogos, Manuel. 2007. Ikone und Witzfigur. Die jiddische Mamme: eine kulturelle Karriere. Jüdische Allgemeine http://www.juedische-allgemeine.de/article/ view/id/3941 (14. Juni 2014) 
Günter, Manuela. 2000. Identität und Identifizierung. Einige Überlegungen zur Konstruktion des „Juden“ nach dem Holocaust. In: Dochartaigh O', Pol (Hrsg.): Jews in German Literature since 1945: German-Jewish Literature? AmsterdamAtlanta, Rodopi, 435-446.

Herzig, Arno. 2005. Jüdische Geschichte in Deutschland. Von den Anfängen bis zur Gegenwart. Bonn, Lizensausgabe für die Bundeszentrale für politische Bildung.

Hofmann, Michael. 1998. Erzählen nach Auschwitz in Uwe Johnsons Jahrestagen. In: Braese, Stephan-Gehle, Holger-Kiesel, Doron-Loewy, Hanno (Hrsg.): Deutsche Nachkriegsliteratur und der Holocaust. Frankfurt am Main-New York: Campus, 197-212.

Horch, Hans-Otto und Shedletzky, Itta. 1992. Die deutsch-jüdische Literatur und ihre Geschichte. In: Schoeps, Julius H. (Hrsg.): Neues Lexikon des Judentums. Gütersloh-München, Bertelsmann Lexikon Verlag, 291. http://www.zentralratdjuden.de/de/topic/5.html (13. Juni 2014)

Kühl, Inga-Marie. 2001. Zwischen Trauma, Traum, und Tradition: Identitätskonstruktionen in der jungen jüdischen Gegenwartsliteratur. Dissertation zur Erlangung des akademischen Grades Doctor Philosophiae an der Philosophischen Fakultät II der Humboldt-Universität zu Berlin.

Lamping, Dieter. 1998. Von Kafka bis Celan. Jüdischer Diskurs in der deutschen Literatur des 20. Jahrhunderts. Göttingen, Vandenhoeck und Ruprecht.

Lilli, Waldemar. 1982. Grundlagen der Stereotypisierung. Göttingen, Verlag für Psychologie.

Ludewig, Anna-Dorothea. 2008. „Schönste Heidin, süßeste Jüdin!“ Die „Schöne Jüdin“ in der europäischen Literatur zwischen dem 17. und 19. Jahrhundert ein Querschnitt. Medaon 3/2008

Ludewig, Anna-Dorothea. 2011. There is More to Being a Jewish Mother than Being Jewish and a Mother. Remarks on the image of the Jewish Mother in the 20th century. Vortrag an der Freien Universität Berlin http://holylit.wordpress. com/2010/11/16/image-of-the-jewish-mother/ (14. Juni 2014)

Mache, Beata. 2004. Polenbilder in der deutsch-jüdischen Gegenwartsprosa. Magisterarbeit. Duisburg-Essen, Universität.

Oişteanu, Andrei. 2005. Imaginea evreului în cultura română. Cluj, Kriterion.

Prangel, Matthias: Die Grundangst der Juden in Deutschland. Gespräch mit Rafael Seligmann. http://www.literaturkritik.de/public/rezension.php?rez_id=6370 (14. Juni 2014)

Robertson, Ritchie. 2004. „Rafael Seligmann’s Rubinsteins Versteigerung. The German-Jewish Family Novel before and after the Holocaust“. In: Bloom, Harold (Hrsg.): Literature of the Holocaust, 237-256. Broomall, PA, Chelsea House Publishers. 
Schmitz, Walter. 2006. „Dem Rafi sein Kampf...“ Das Identitätstrauma des Juden in der deutschen Diaspora in Film und epischer Prosa von Rafael Seligmann. In: Zabel, Hermann (Hrsg.): Stimmen aus Jerusalem: zur deutschen Sprache und Literatur in Palästina/Israel. Berlin, LIT Verlag, 370-411.

Schoeps, Julius H.: Deutsche - und nichts anderes 1992. Vom Patriotismus deutscher Juden. Spiegel Spezial 2. 94-112.

Schruff, Helene. 2000. Wechselwirkungen: deutsch-jüdische Identität in erzählender Prosa der 'Zweiten Generation'. Hildesheim, Olms Verlag.

Seligmann, Rafael. 1992. Die Juden leben. Der Spiegel 1992. 75-78.

Seligmann, Rafael. 1997. Mut der Meshuggenen. Der Spiegel 23. 211-213.

Seligmann, Rafael. 2000 [1990]. Die jiddische Mamme. München, DTV.

Steinecke, Hartmut. 2000. Deutsch-jüdische Literatur der „zweiten Generation“ und die Wende: „Geht jetzt wieder alles von vorne los?“ In: Wehdeking, Volker (Hrsg.): Mentalitätswandel in der deutschen Literatur zur Einheit (19902000)... Berlin, Erich Schmidt, 189-200.

Wirtz, Susanne. 2011. Jüdische Autoren der Gegenwart. Probleme - Positionen - Themen. Tribüne. Zeitschrift zum Verständnis des Judentums, 50. Jahrgang, Heft 198, 152-160. 